\title{
Primary CNS Lymphoma in the Elderly: The Challenge
}

\author{
Tali Siegal $^{\mathrm{a}}$ Osnat Bairey ${ }^{\mathrm{b}, \mathrm{c}}$ \\ ${ }^{a}$ Neuro-Oncology Center, Davidoff Cancer Center, Rabin Medical Center - Beilinson Hospital, Petach Tikva, Israel; \\ ${ }^{\mathrm{b}}$ Institute of Hematology, Davidoff Cancer Center, Rabin Medical Center - Beilinson Hospital, Petach Tikva, Israel; \\ 'Sackler Faculty of Medicine, Tel Aviv University, Tel Aviv, Israel
}

\section{Keywords}

Autologous stem cell transplantation - Chemotherapy ·

Clinical trials · Elderly $\cdot$ High-dose methotrexate $\cdot$ Primary

CNS lymphoma · Survival · Toxicity

\begin{abstract}
Primary central nervous system (CNS) lymphoma is an aggressive brain tumor sensitive to chemotherapy and radiotherapy. Its incidence has increased in the elderly, and they account for the majority of patients. The median survival of patients older than 70 years did not change over the last 40 years and remained in the range of 6-7 months. The definition of elderly is nonuniform, and chronological age is not the best marker of treatment tolerability or a predictor of treatment-related toxicity. Some patients who are fit can tolerate induction, consolidation, and even high-dose chemotherapy with autologous stem cell transplantation, whereas others who have multiple comorbidities with reduced renal and bone marrow function can tolerate only intermediate doses of methotrexate. The latter may benefit from maintenance treatment. The "elderly" are also susceptible to the accelerated and detrimental cognitive side effects of wholebrain irradiation which is an alternative consolidation to high-dose chemotherapy. The optimal treatment remains an unresolved matter. A comprehensive comorbidity and geriatric assessment is imperative for appraisal of treatment-in-
\end{abstract}

\section{KARGER}

(c) 2019 S. Karger AG, Basel

E-Mail karger@karger.com

www.karger.com/aha duced risks for CNS and systemic toxicity. An individualized approach is required aiming to prolong survival while minimizing toxicity. Future studies should assess the potential of new agents for improving outcome and maintaining quality of life.

(c) 2019 S. Karger AG, Basel

\section{Introduction}

Elderly patients account for the majority of primary central nervous system (CNS) lymphomas (PCNSL), a disease classified as highly aggressive non-Hodgkin lymphoma confined to the CNS. A recent study revealed that the incidence of PCNSL increased from 0.1 per 100,000 in the 1970 s to 0.4 per 100,000 in the 1980 s, correlating with an increase in the diagnosis of patients $\geq 70$ years [1]. The incidence in elderly patients aged $70-79$ years is 4.32 compared to 0.08 in the 3 rd decade of life. Although PCNSL often responds to initial treatment, survival is inferior when compared to lymphomas located outside the $\mathrm{CNS}$, and despite the fact that the median overall survival (OS) of all patients has doubled over the last 40 years (from 12.5 to 25 months), this survival benefit has remained limited to patients younger than 70 years [1]. The devastating fact is that median survival in the elderly population (aged 70 years or more) has not changed during 
this period and remained in the range of 6-7 months. This poor outcome highlights the need to anticipate factors that contribute to treatment failure in order to improve treatment strategies when targeting the elderly population. Recognition of aspects such as comorbidities and risks of treatment-induced CNS and systemic toxicity is essential for successful treatment planning and for the design of future clinical trials. In this review, we outline main issues related to the delay in diagnosis, choice of therapeutic modalities, and factors related to PCNSL treatment failure in the elderly.

\section{How to Define "Elderly" PCNSL Patients}

The definition of elderly is nonuniform in the medical literature. In systemic diffuse large B-cell lymphomas, it has shifted over the years to a more advanced level of age, i.e., above the common cutoff age of 60 years. In PCNSL, studies that assessed prognostic factors have consistently correlated older age (over 50 or 60 years) with poorer outcome, and age of 60 years and over was also associated with a higher risk of neurotoxic effects of chemoradiation [2]. Consequently, 60 years of age is frequently used as a cutoff to define the elderly population of PCNSL patients. Nevertheless, several studies have shown that fit patients above the age of 60 years can tolerate high-dose chemotherapy and even an intensive up-front treatment with a high overall response rate reported (range: 60-89\%) including a proportion of long-term survivors (Table 1) [317]. Yet, many clinical trials exclude patients above the age of 70 years $[11,18]$, and about a quarter of PCNSL patients older than 70 years who survive more than 3 months from diagnosis do not receive chemotherapy at all [19]. The fraction of patients who have not been treated with chemotherapy increased from 14 to 23 , and to $44 \%$ in the age groups of $61-70,71-80$, and $>80$ years, respectively. With the recognition that high-dose methotrexate (HD-MTX)-based treatment may provide survival benefit for older individuals with adequate organ function, the number of elderly patients not treated with chemotherapy tends to decrease over time, particularly in academic research facilities. Still, analysis of characteristics and outcome of patients with PCNSL in a "real-life" setting compared to a clinical trial indicated that the outcome of PCNSL patients treated outside clinical trials was poor probably due to more unfavorable prognostic factors [20]. Thus, outcomes of expert management provided for this rare disease in clinical trials pertain for selected subgroups of elderly patients who are relatively younger

Primary CNS Lymphoma in the Elderly and fit. More so, inconsistencies in the definition of "elderly" patients complicate the interpretation of many studies, particularly because almost all published clinical trials do not provide analysis of treatment outcome which is stratified by age groups (e.g., outcome for the age group 60-69 vs. 70-79 years). In reality, most clinical trials and treating centers tend to regard PCNSL patients up to age 70 years as candidates for intensive up-front therapy while reserving less aggressive approaches for those above the age of 70 years. Yet age alone is not the ultimate aspect by which a treatment approach can be tailored to a patient, and other intervening factors should be taken into account.

\section{Elderly Patients Have Greater Comorbidities}

Age alone is not a reliable criterion for deciding on PCNSL treatment since there is a great heterogeneity even among patients of the same age. Older age is associated with an accumulation of physiological deficits that alter the pharmacokinetics and pharmacodynamics of therapy and may increase the risk of toxicities (nephrotoxicity, hepatotoxicity, myelosuppression, and neurotoxicity). Functional status, both at the time of diagnosis and the premorbid status, should be considered as important predictors of OS and drug toxicity. Multiple parameters, including clinical assessment, performance status, comorbidities, functional reserve, and tolerance to drug toxicity, should be accounted for when choosing treatment for the elderly PCNSL patient.

The American Society of Clinical Oncology (ASCO) guideline advocates use of geriatric assessment tools to identify vulnerabilities not routinely captured in oncology assessments for patients aged 65 years or older who receive chemotherapy [21]. The use of geriatric assessment tools should help identify nononcological problems amenable to intervention. These tools are useful for prediction of chemotherapy toxicity and mortality [22]. A decreased functional reserve may increase the susceptibility to chemotherapy-related complications but, as many of these impairments are reversible in PCNSL, they should not prevent administration of chemotherapy or HD-MTX. Patients with PCNSL often experience acute deterioration of aspects that are examined in the geriatric assessment, such as motor function, cognitive deterioration, depression, and frequent falls. These are manifestations of CNS involvement, and, therefore, the regular geriatric assessment may be less relevant to PCNSL. It is suggested that an adaptive new geriatric assessment may 
Table 1. Studies on first-line chemotherapy in elderly PCNSL patients published between 2000 and 2018

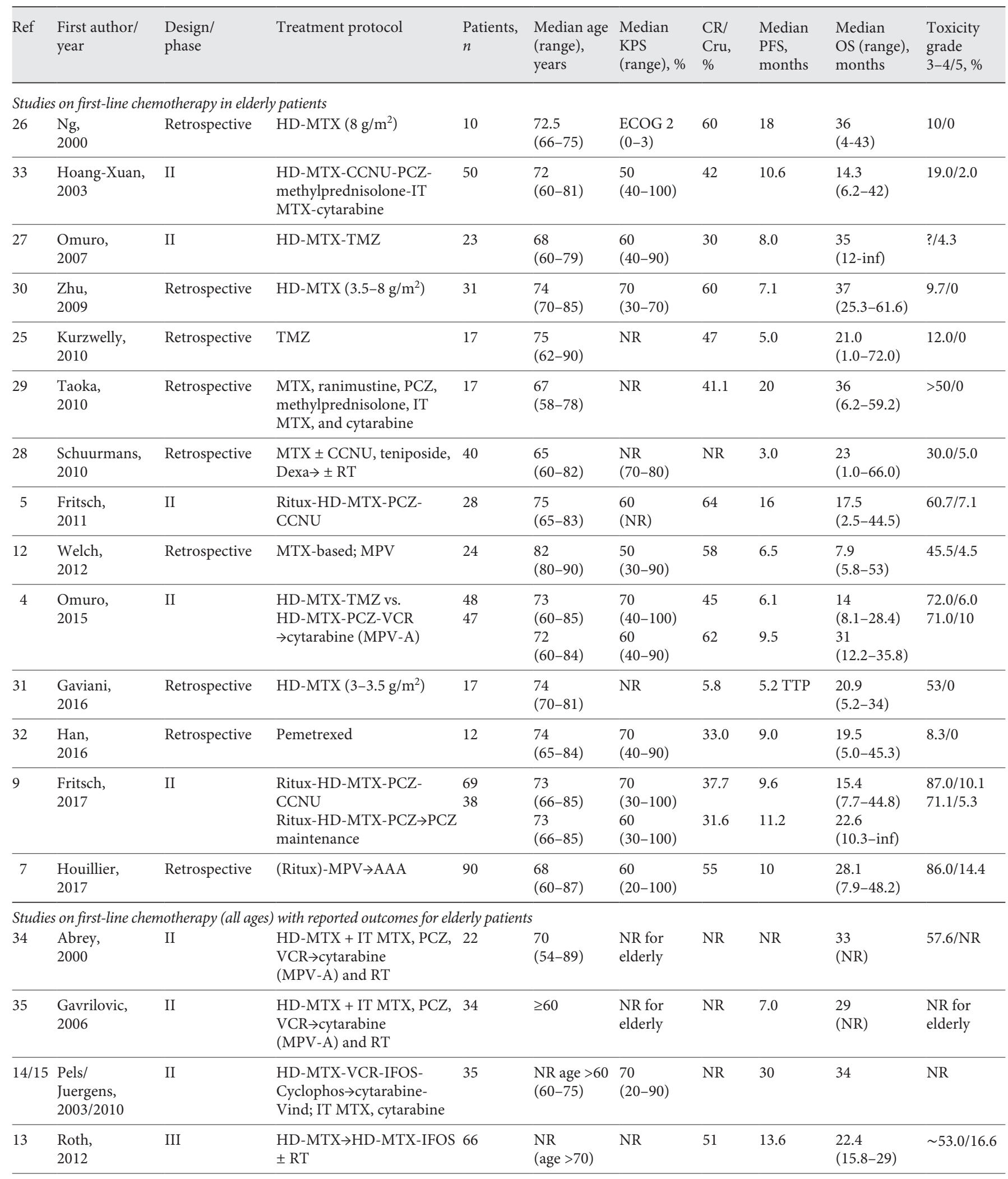


Table 1 (continued)

\begin{tabular}{|c|c|c|c|c|c|c|c|c|c|c|}
\hline Ref & $\begin{array}{l}\text { First author/ } \\
\text { year }\end{array}$ & $\begin{array}{l}\text { Design/ } \\
\text { phase }\end{array}$ & Treatment protocol & $\begin{array}{l}\text { Patients, } \\
n\end{array}$ & $\begin{array}{l}\text { Median age } \\
\text { (range), } \\
\text { years }\end{array}$ & $\begin{array}{l}\text { Median } \\
\text { KPS } \\
\text { (range), \% }\end{array}$ & $\begin{array}{l}\mathrm{CR} / \\
\mathrm{Cru} \\
\%\end{array}$ & $\begin{array}{l}\text { Median } \\
\text { PFS, } \\
\text { months }\end{array}$ & $\begin{array}{l}\text { Median } \\
\text { OS (range), } \\
\text { months }\end{array}$ & $\begin{array}{l}\text { Toxicity } \\
\text { grade } \\
3-4 / 5, \%\end{array}$ \\
\hline 16 & $\begin{array}{l}\text { Pulczynski, } \\
2015\end{array}$ & II & $\begin{array}{l}\text { Ritux-HD-MTX- } \\
\text { TMZ } \rightarrow \text { cytarabine-Vind: } \\
\text { IT DepoCyte; } \rightarrow \text { TMZ } \\
\text { maintenance } 12 \text { mo }\end{array}$ & 27 & $\begin{array}{l}70 \\
(66-75)\end{array}$ & ECOG 2 & 69.2 & $\begin{array}{l}\text { 2-year PFS } \\
44.1 \%\end{array}$ & $\begin{array}{l}\mathrm{NR} \\
>22\end{array}$ & $89.1 / \mathrm{NR}$ \\
\hline 3 & $\begin{array}{l}\text { Schorb, } \\
2017\end{array}$ & Retrospective & $\begin{array}{l}\text { HD-thiotepa-based } \\
\text { chemoTx + ASCT }\end{array}$ & 15 & $\begin{array}{l}70 \\
(66-77)\end{array}$ & $\begin{array}{l}65 \\
(30-90)\end{array}$ & 69 & 51.5 & 122 & NR \\
\hline
\end{tabular}

ASCT, autologous stem cell transplantation; CCNU, lomustine; chemoTx., chemotherapy; CR, complete response; Cru, unverified CR; Cyclophos, cyclophosphamide; Dexa, dexamethasone; EA, etoposide-cytarabine; HD-MTX, high-dose methotrexate; IFOS, ifosphamide; IT, intrathecal; MPV, MTXPCZ-vincristine; MPV-A, MTX-PCZ-vincristine-cytarabine; NR, not reported; OS, overall survival; PCZ, procarbazine; PFS, progression-free survival; Ref, reference; Ritux, rituximab; RT, radiotherapy; TMZ, temozolomide; TTP, time to progression; VCR, vincristine; Vind, vindesine.

be needed for this malignancy as the acute and severe neurocognitive and functional deteriorations are often reversible with response to therapy. So far, geriatric assessment tools have not been applied prospectively to older PCNSL patients, and their utility for clinical application should be assessed in the future. Collaboration with caregivers is another essential aspect needed for the implement of geriatric-assessment-guided interventions.

Older patients are usually offered less aggressive and maybe less effective treatment modalities. Suboptimal treatment secondary to age may be partly responsible for a worse outcome. Notably, a "fit elderly" individual may tolerate standard doses and schedules of chemotherapeutic medications and thus may obtain the same benefit as younger patients. A model that has been developed (CRASH score) considers the specific chemotherapy regimen to be used, laboratory values, and assessments of functional, mental, and nutritional status [22]. Routine use of such scores may augment the fraction of elderly patients offered optimal treatment.

Treatment of PCNSL includes induction and consolidation phases. HD-MTX is the single most important agent for induction of response in all age groups. In elderly patients, the use of HD-MTX may require dose adjustments because of reduced creatinine clearance and limited bone marrow reserve. A study that prospectively evaluated toxicity of MTX ( $4 \mathrm{mg} / \mathrm{m}^{2}$ per treatment cycle) which was administered to 110 elderly PCNSL patients found that toxicity was generally mild [23]. However, dose reduction was required to adapt to low glomerular filtration rate in $18 \%$ of patients aged $\leq 60$ years and in
$44 \%$ of those older than 60 years. The difference between these age groups was statistically significant $(p=0.001)$. Grade 3-4 toxicity occurred in less than $10 \%$, and both incidence and severity of the toxicity were similar in all age groups. Therapy discontinuation owing to MTX toxicity also did not differ by age and occurred in $5-8 \%$. So, it seems that HD-MTX treatment is safe for PCNSL patients regardless of age, with adherence to dose reduction adapted to kidney function. The impact of dose reduction on treatment efficacy remains unclear.

In addition to frequent dose MTX reductions, the treatment of elderly PCNSL patients is further hampered by their high vulnerability to more toxic and particularly myelosuppressive agents included in MTX-based multidrug protocols (Table 1). The increased susceptibility also accounts for consolidation treatment, which is based on either whole-brain radiotherapy (WBRT) or on highdose chemotherapy with or without autologous stem cell transplantation (ASCT). As a result, the induction chemotherapy is of utmost importance since many elderly patients are not candidates for therapeutic modalities currently used for consolidation.

\section{Diagnosis and Treatment of Elderly Patients with PCNSL}

\section{Diagnosis}

Signs and symptoms of PCNSL are nonspecific. Frequent presentations (in 50-70\% of cases) include personality changes and cognitive impairment, which are often 
attributed to other common disorders associated with mental deterioration in older age. Focal neurological deficits affect half of the patients, and it is frequently mistaken early on as a cerebrovascular disorder. Therefore, delay in diagnosis might be a factor contributing to a poor functional status at the time of diagnosis. Contrast-enhanced magnetic resonance imaging typically shows intense contrast-enhanced lesion(s) with significant diffusion restriction due to high cell density which is often suggestive of the diagnosis. Nonetheless, imaging alone does not allow discrimination from other malignant tumors or inflammatory lesions, and tissue diagnosis should be obtained without delay.

\section{Treatment}

There is still no standard treatment for PCNSL largely due to the low incidence of the disease, which makes it challenging to conduct large randomized studies. PCNSL is sensitive to radiation and chemotherapy. Nonetheless, the treatment approach has changed significantly over the past few decades shifting towards chemotherapy-only once the high incidence of treatment-induced neurotoxicity was realized [24]. Radiation-induced neurotoxicity is observed more frequently in the elderly and results in progressive severe cognitive dysfunction, ataxia, and urinary incontinence. Neurotoxicity occurred in $19-83 \%$ of patients older than 60 years who received WBRT following MTX-based chemotherapy. Furthermore, it tends to occur more severely and rapidly with advancing age at the time of treatment. Attempts to reduce neurotoxicity, without impairing outcomes, prompted the exploration of chemotherapy-only treatment regimens, but the number of studies dedicated to elderly PCNSL patients remains limited (Table 1). Consequently, the optimal management of elderly patients is still poorly defined. There is a lack of prospectively collected data to guide treatment decisions in this patient population.

Table 1 summarizes the reported outcomes of firstline chemotherapy in elderly PCNSL patients. Fourteen studies were dedicated to elderly patients $[4,5,7,9,12$, 24-33], and 7 studies accrued adult patients of all ages but contained information on outcome of the elderly subgroup [6, 13-16, 34, 35]. One retrospective study [3] described the outcome of thiotepa-based chemotherapy with ASCT administered to 52 elderly lymphoma patients, of whom 15 were PCNSL treated by this protocol as first-line approach. Nine of the 14 studies dedicated to elderly patients $[7,12,25,26,28-32]$ were retrospective, and 4 were phase II studies $[4,5,9,27]$, with one of them designed as a randomized phase II study [4]. Six studies that reported on elderly subgroups were phase II studies $[6,14-16,34,35]$, and 1 was a retrospective analysis of elderly patients in a phase III study [13]. Treatment regimens varied from monotherapy to multidrug HD-MTXbased combinations. Later on, rituximab has been added to chemotherapy regimens with the hope to improve outcome of PCNSL similarly to the added positive effect obtained in systemic B-cell lymphomas. However, up to date, it remains unclear whether rituximab improves outcome in PCNSL or whether it augments immunosuppression particularly in the elderly, vulnerable population.

The rate of complete responses (CR) across all studies of the elderly is ranging from 30 to $69 \%$, which is similar to the reported CR rate of all age groups observed in a 3-arm randomized multicenter study that compared 2-, 3-, and 4-drug combinations of intensive HD- MTX-based chemotherapy protocols (HD-MTXcytarabine, HD-MTX-cytarabine plus rituximab, or HD-MTX-cytarabine-rituximab plus thiotepa) [11]. More so, it seems that the use of less toxic regimens such as single-agent HD-MTX or the MTX-procarbazine-vincristine (MPV) with or without cytarabine consolidation is as effective in obtaining the same CR rate. Similar observations have been noted in a meta-analysis of 741 immunocompetent patients aged $\geq 60$ years with newly diagnosed PCNSL. No difference in survival was found between patients treated with either HD-MTX plus oral alkylating agents or with more intensive intravenous combinations [36].

Median progression-free survival (PFS) and OS in Table 1 show that OS is ranging from 7.9 to 36 months, and it is much longer than the relatively short PFS. This probably reflects the effect of salvage therapy (such as WBRT) that prolongs survival beyond progression regardless of its negative impact on quality of life. Notably, outcomes of published treatment series do not reflect the "real-life" experience where median OS of the elderly remained in the range of 6-7 months $[1,19]$. However, HDMTX is relatively safe treatment for PCNSL patients regardless of age [23], and even patients aged 80 years or more can tolerate the HD-MTX regimen (such as MPV) [12]. Up to $20 \%$ of all PCNSL patients are aged 80 years or older, and this population is growing rapidly. This older subgroup of patients should not be denied treatment solely on the basis of age as those who respond to the induction regimen gain substantial benefit in terms of performance status and survival, while those who do not respond to induction therapy are unlikely to improve. 


\section{Consolidation Therapy}

Consolidation therapy is given after the induction phase to those patients who achieve complete or partial responses. It is still unclear whether consolidation improves disease control or survival of the subgroup of patients eligible for it. There are 3 modalities of consolidation: WBRT, nonmyeloablative high-dose chemotherapy, and myeloablative conditioning with ASCT. In older patients, consolidation with WBRT has a deleterious effect, and it compromises survival because of neurotoxic effects [24]. Therefore, consolidation regimens such as high-dose chemotherapy may be a more reasonable option, but the major obstacles for this approach are the advanced age and poor general condition of most patients. Consequently, consolidation is given to selected fit patients. Table 1 shows that cytarabine was the main agent that served for consolidation in the elderly $[4,7,14,15,34,35]$. One study retrospectively evaluated whether intensified consolidation with three 28 -day cycles of cytarabine $\left(3 \mathrm{~g} / \mathrm{m}^{2}\right.$ days 1-2) improved outcome in patients older than 60 years [7]. Ninety patients were included. They received the MPV induction regimen that was followed by intensified cytarabine treatment (MPV-AAA). Outcomes were compared with the historical single cytarabine cycle (MPV-A). The conclusion was that the intensified cytarabine regimen does not improve the efficacy in the elderly but increases toxicity. Notably, the rate of treatment-related death amounted to $14.4 \%$. Another phase II study used an intensive chemotherapy protocol that included consolidation with high-dose etoposide plus cytarabine [6]. The study accrued 44 patients of all ages, with 23 of them aged $>60$ years. The conclusion was that dose-intensive consolidation is feasible and yields rates of PFS and OS at least comparable to those regimens involving WBRT. It is mentioned that patients aged $>60$ years did as well as younger patients, but the exact figures of median PFS and OS of the older subgroup are not specified.

\section{High-Dose Chemotherapy with ASCT}

This approach represents an effective treatment option for selected refractory and relapsed PCNSL patients [37]. The conditioning regimen usually consists of thiotepa-based combinations (including BCNU and cyclophosphamide). Its role as consolidation therapy for patients of all ages is still under continuous evaluation (IELSG 32-NCT 01011920; IELSG 43-NCT02531841; PRECIS-NCT 00863460). Because of the high risk and associated toxicity, this treatment is usually offered to younger patients ( $<65$ years) with a good performance status. Schorb et al. [3] reported retrospectively on the outcome of 52 patients with PCNSL aged $\geq 65$ years who received thiotepa-based conditioning with ASCT ( $29 \%$ as first-line treatment). The conclusion was that in selected elderly patients, this is an effective, safe treatment if conducted at experienced centers.

\section{Maintenance Treatment}

In the elderly who cannot tolerate consolidation therapy, maintenance treatment may serve as a feasible alternative approach. There are several available and promising chemotherapeutic and targeted agents that may play a role in maintenance treatment of PCNSL (e.g., procarbazine, temozolomide, lenalidomide, and ibrutinib) [38], but their effectiveness has to be studied in a prospective design. Maintenance therapy may delay relapses and maintain tumor dormancy when administered as metronomic chemotherapy or targeted therapy. However, owing to the investigational nature of these approaches in PCNSL and the current lack of proven efficacy of the available drugs, all maintenance regimens are highly empirical in terms of optimal dose and schedule. Today, there is no randomized clinical trial in PCNSL proving that maintenance treatment is of benefit, and, therefore, it is currently an experimental approach. There are several ongoing or planned studies that aim to evaluate maintenance therapy in elderly patients: a phase II study of ibrutinib (NCT02623010), a phase III study of temozolomide maintenance versus observation (NCT02313389), and a not-yet-recruiting randomized phase II study that aims to compare lenalidomide and procarbazine as maintenance modalities (NCT03495960).

\section{Treatment-Related Toxicity}

Treatment-related toxicity may be directly induced by chemotherapy (e.g., nephrotoxicity, hepatotoxicity, and myelosuppression), and as such it is recognized relatively early after drug administration. Neurotoxicity, on the other hand, may develop during the course of a successful treatment or afterwards, and its identification is, therefore, delayed. Table 1 lists the reported rates of grade 3-4 treatment-related toxicity of the various therapeutic regimens as well as the rate of treatment-related death (grade 5). The use of monotherapy seems to be associated with a substantially lower rate of toxicity and with no treatmentrelated death $[25,26,31,32]$, while multidrug treatment increases the rate of grade 3-4 toxicity once myelosuppressive agents are added. The more intensive regimens are associated with severe toxicity (81-89\%). Not surprisingly, the rate of treatment-related death increases as well (ranging from 2 to $14.4 \%$ ). 
Neurotoxicity is observed more frequently in the elderly and may be related to either chemotherapy alone, WBRT, or to the combined use of these modalities. HDMTX is a known cause of leukoencephalopathy, and if WBRT is given following MTX-based chemotherapy signs and symptoms are recognized at an average delay of 3-52 months after completing treatment and earlier in patients older than 60 years [24]. Radiation-induced neurotoxicity tends to increase over time, and, therefore, it is more pronounced in those who survive for longer periods due to an effective treatment. On the other hand, subtle cognitive changes often associated with chemotherapy are likely underestimated because they require routine neurocognitive testing, and it remains unclear to what degree quality of life is compromised.

\section{Conclusions}

The optimal treatment for PCNSL is an unresolved matter, especially regarding the management of elderly patients who comprise the majority of patients with this infrequent disease. The challenges are numerous. An un- certain definition of "elderly" is an important cause of uncertainty because chronological age may not be the best marker of tolerability of treatment or a predictor of treatment-related toxicity. There is great variability in the functional status and comorbidities within this group of patients aged 60 years or more. Many of them tolerate HD-MTX without difficulty, and MTX remains the single most important agent in all effective treatment regimens. In the elderly, treatment approaches differ from those in the young. In the younger patients, the intent is curative, and the price of intensive and toxic treatment necessary to achieve this goal is unacceptable for the vast majority of elderly patients. Yet, a subgroup of fit elderly patients may still tolerate it. Therefore, future studies need to focus on stratification of the elderly with the goal of achieving and maintaining remission, improving tolerability, minimizing treatment-induced toxicity, and preserving function and quality of life. These objectives entail a more individualized approach aimed to prolong PFS and OS while minimizing toxicity and preserving function. Hopefully, future studies with multi-institutional and multidisciplinary efforts will assess the potential of new agents for improving outcome and maintaining quality of life.

\section{References}

1 Mendez JS, Ostrom QT, Gittleman H, Kruchko C, DeAngelis LM, Barnholtz-Sloan JS, et al. The elderly left behind-changes in survival trends of primary central nervous system lymphoma over the past 4 decades. Neuro Oncol. 2018 Apr 9;20(5):687-694.

2 Hoang-Xuan K, Bessell E, Bromberg J, Hottinger AF, Preusser M, Rudà R, et al; European Association for Neuro-Oncology Task Force on Primary CNS Lymphoma. Diagnosis and treatment of primary CNS lymphoma in immunocompetent patients: guidelines from the European Association for NeuroOncology. Lancet Oncol. 2015 Jul;16(7):e32232.

3 Schorb E, Fox CP, Fritsch K, Isbell L, Neubauer A, Tzalavras A, et al. High-dose thiotepa-based chemotherapy with autologous stem cell support in elderly patients with primary central nervous system lymphoma: a European retrospective study. Bone Marrow Transplant. 2017 Aug;52(8):1113-9.

4 Omuro A, Chinot O, Taillandier L, Ghesquieres $\mathrm{H}$, Soussain C, Delwail V, et al. Methotrexate and temozolomide versus methotrexate, procarbazine, vincristine, and cytarabine for primary CNS lymphoma in an elderly population: an intergroup ANOCEF-GOELAMS randomised phase 2 trial. Lancet Haematol. 2015 Jun;2(6):e251-9.
5 Fritsch K, Kasenda B, Hader C, Nikkhah G, Prinz M, Haug V, et al. Immunochemotherapy with rituximab, methotrexate, procarbazine, and lomustine for primary CNS lymphoma (PCNSL) in the elderly. Ann Oncol. 2011 Sep;22(9):2080-5.

6 Rubenstein JL, Hsi ED, Johnson JL, Jung SH, Nakashima MO, Grant B, et al. Intensive chemotherapy and immunotherapy in patients with newly diagnosed primary CNS lymphoma: CALGB 50202 (Alliance 50202). J Clin Oncol. 2013 Sep;31(25):3061-8.

7 Houillier C, Ghesquières $\mathrm{H}$, Chabrot C, Soussain C, Ahle G, Choquet S, et al. Rituximab, methotrexate, procarbazine, vincristine and intensified cytarabine consolidation for primary central nervous system lymphoma (PCNSL) in the elderly: a LOC network study. J Neurooncol. 2017 Jun;133(2):315-20.

8 Ferreri AJ, Cwynarski K, Pulczynski E, Fox CP, Schorb E, La Rosée P, et al; International Extranodal Lymphoma Study Group (IELSG). Whole-brain radiotherapy or autologous stem-cell transplantation as consolidation strategies after high-dose methotrexate-based chemoimmunotherapy in patients with primary CNS lymphoma: results of the second randomisation of the International Extranodal Lymphoma Study Group-32 phase 2 trial. Lancet Haematol. 2017 Nov;4(11):e510-23.
9 Fritsch K, Kasenda B, Schorb E, Hau P, Bloehdorn J, Möhle R, et al. High-dose methotrexate-based immuno-chemotherapy for elderly primary CNS lymphoma patients (PRIMAIN study). Leukemia. 2017 Apr;31 (4):846-52.

10 Korfel A, Thiel E, Martus P, Möhle R, Griesinger F, Rauch M, et al. Randomized phase III study of whole-brain radiotherapy for primary CNS lymphoma. Neurology. 2015 Mar;84(12):1242-8.

11 Ferreri AJ, Cwynarski K, Pulczynski E, Ponzoni M, Deckert M, Politi LS, et al; International Extranodal Lymphoma Study Group (IELSG). Chemoimmunotherapy with methotrexate, cytarabine, thiotepa, and rituximab (MATRix regimen) in patients with primary CNS lymphoma: results of the first randomisation of the International Extranodal Lymphoma Study Group-32 (IELSG32) phase 2 trial. Lancet Haematol. 2016;3(5):e217-27.

12 Welch MR, Omuro A, Deangelis LM. Outcomes of the oldest patients with primary CNS lymphoma treated at Memorial SloanKettering Cancer Center. Neuro Oncol. 2012 Oct;14(10):1304-11.

13 Roth P, Martus P, Kiewe P, Möhle R, Klasen H, Rauch $\mathrm{M}$, et al. Outcome of elderly patients with primary CNSlymphoma in the G-PCNSLSG-1 trial. Neurology. 2012 Aug;79(9):890-6. 
14 Pels H, Schmidt-Wolf IG, Glasmacher A, Schulz H, Engert A, Diehl V, et al. Primary central nervous system lymphoma: results of a pilot and phase II study of systemic and intraventricular chemotherapy with deferred radiotherapy. J Clin Oncol. 2003 Dec;21(24): 4489-95.

15 Juergens A, Pels H, Rogowski S, Fliessbach K, Glasmacher A, Engert A, et al. Long-term survival with favorable cognitive outcome after chemotherapy in primary central nervous system lymphoma. Ann Neurol. 2010 Feb; 67(2):182-9.

16 Pulczynski EJ, Kuittinen O, Erlanson M, Hagberg $\mathrm{H}$, Fosså A, Eriksson M, et al. Successful change of treatment strategy in elderly patients with primary central nervous system lymphoma by de-escalating induction and introducing temozolomide maintenance: results from a phase II study by the Nordic Lymphoma Group. Haematologica. 2015 Apr;100(4):534-40.

17 Abrey LE, Ben-Porat L, Panageas KS, Yahalom J, Berkey B, Curran W, et al. Primary central nervous system lymphoma: the Memorial Sloan-Kettering Cancer Center prognostic model. J Clin Oncol. 2006 Dec;24(36):5711-5.

18 Schorb E, Finke J, Ferreri AJ, Ihorst G, Mikesch K, Kasenda B, et al. High-dose chemotherapy and autologous stem cell transplant compared with conventional chemotherapy for consolidation in newly diagnosed primary CNS lymphoma - a randomized phase III trial (MATRix). BMC Cancer. 2016 Apr;16(1):282.

19 Fallah J, Qunaj L, Olszewski AJ. Therapy and outcomes of primary central nervous system lymphoma in the United States: analysis of the National Cancer Database. Blood Adv. 2016 Nov;1(2):112-21.

20 Zeremski V, Koehler M, Fischer T, Schalk E. Characteristics and outcome of patients with primary CNS lymphoma in a "real-life" setting compared to a clinical trial. Ann Hematol. 2016 Apr;95(5):793-9.
21 Mohile SG, Dale W, Somerfield MR, Schonberg MA, Boyd CM, Burhenn PS, et al. Practical Assessment and Management of Vulnerabilities in Older Patients Receiving Chemotherapy: ASCO Guideline for Geriatric Oncology. J Clin Oncol. 2018 Aug;36(22): 2326-47.

22 Extermann M, Boler I, Reich RR, Lyman GH, Brown RH, DeFelice J, et al. Predicting the risk of chemotherapy toxicity in older patients: the Chemotherapy Risk Assessment Scale for High-Age Patients (CRASH) score. Cancer. 2012 Jul;118(13):3377-86.

23 Jahnke K, Korfel A, Martus P, Weller M, Herrlinger U, Schmittel A, et al; German Primary Central Nervous System Lymphoma Study Group (G-PCNSL-SG). High-dose methotrexate toxicity in elderly patients with primary central nervous system lymphoma. Ann Oncol. 2005 Mar; 16(3):445-9.

24 Daras M, DeAngelis LM. Management of elderly patients with primary central nervous system lymphoma. Curr Neurol Neurosci Rep. 2013 May; 13(5):344.

25 Kurzwelly D, Glas M, Roth P, Weimann E, Lohner H, Waha A, et al. Primary CNS lymphoma in the elderly: temozolomide therapy and MGMT status. J Neurooncol. 2010 May; 97(3):389-92.

26 Ng S, Rosenthal MA, Ashley D, Cher L. Highdose methotrexate for primary CNS lymphoma in the elderly. Neuro Oncol. 2000 Jan;2(1): $40-4$

27 Omuro AM, Taillandier L, Chinot O, Carnin C, Barrie M, Hoang-Xuan K. Temozolomide and methotrexate for primary central nervous system lymphoma in the elderly. J Neurooncol. 2007 Nov;85(2):207-11.

28 Schuurmans M, Bromberg JE, Doorduijn J, Poortmans P, Taphoorn MJ, Seute T, et al. Primary central nervous system lymphoma in the elderly: a multicentre retrospective analysis. Br J Haematol. 2010 Oct;151(2):179-84.

29 Taoka K, Okoshi Y, Sakamoto N, Takano S, Matsumura A, Hasegawa Y, et al. A nonradiation-containing, intermediate-dose methotrexate regimen for elderly patients with primary central nervous system lymphoma. Int J Hematol. 2010 Nov;92(4):617-23.
30 Zhu JJ, Gerstner ER, Engler DA, Mrugala MM, Nugent W, Nierenberg K, et al. Highdose methotrexate for elderly patients with primary CNS lymphoma. Neuro Oncol. 2009 Apr;11(2):211-5.

31 Gaviani P, Simonetti G, Innocenti A, Lamperti E, Botturi A, Silvani A. Safety and efficacy of primary central nervous system lymphoma treatment in elderly population. Neurol Sci. 2016 Jan;37(1):131-3.

32 Han S, Wang M, Liu B, Yu J. Pemetrexed for primary central nervous system lymphoma in the elderly. Clin Transl Oncol. 2016 Feb; 18(2):138-43.

33 Hoang-Xuan K, Taillandier L, Chinot O, Soubeyran P, Bogdhan U, Hildebrand J, et al; European Organization for Research and Treatment of Cancer Brain Tumor Group. Chemotherapy alone as initial treatment for primary CNS lymphoma in patients older than 60 years: a multicenter phase II study (26952) of the European Organization for Research and Treatment of Cancer Brain Tumor Group. J Clin Oncol. 2003 Jul;21(14):272631.

34 Abrey LE, Yahalom J, DeAngelis LM. Treatment for primary CNS lymphoma: the next step. J Clin Oncol. 2000 Sep;18(17):3144-50.

35 Gavrilovic IT, Hormigo A, Yahalom J, DeAngelis LM, Abrey LE. Long-term follow-up of high-dose methotrexate-based therapy with and without whole brain irradiation for newly diagnosed primary CNS lymphoma. J Clin Oncol. 2006 Oct;24(28):4570-4.

36 Kasenda B, Ferreri AJ, Marturano E, Forst D, Bromberg J, Ghesquieres $\mathrm{H}$, et al. First-line treatment and outcome of elderly patients with primary central nervous system lympho$\mathrm{ma}$ (PCNSL) - a systematic review and individual patient data meta-analysis. Ann Oncol. 2015 Jul;26(7):1305-13.

37 Citterio G, Reni M, Gatta G, Ferreri AJ. Primary central nervous system lymphoma. Crit Rev Oncol Hematol. 2017 May;113:97-110.

38 Bairey O, Siegal T. The possible role of maintenance treatment for primary central nervous system lymphoma. Blood Rev. 2018 Sep; 32(5):378-86. 